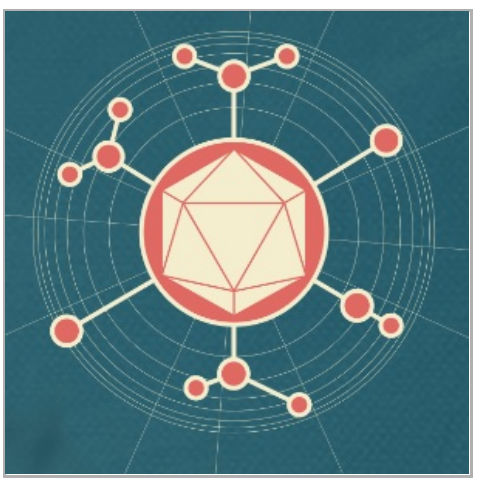

AUG 24, 2020

\section{(3) nCoV-2019 McGill Nextera Flex LibPrep Protocol}

Forked from a private protocol

Marie-Michelle Simon ${ }^{1}$, Sarah J Reiling ${ }^{1}$, Ioannis Ragoussis ${ }^{1}$

${ }^{1}$ McGill University

Coronavirus Method Development Community

McGill Genome Centre

\section{open ठaccess}

\section{DOI:}

dx.doi.org/10.17504/protocol s. io. bjgnkjve

Protocol Citation: MarieMichelle Simon, Sarah J Reiling, loannis Ragoussis 2020.

nCoV-2019 McGill Nextera Flex LibPrep Protocol. protocols.io

https://dx.doi.org/10.17504/p rotocols. io. bjgnkjve

License: This is an open access protocol distributed under the terms of the Creative Commons Attribution License, which permits unrestricted use, distribution, and reproduction in any medium, provided the original author and source are credited

Protocol status: Working We use this protocol and it's working

Created: Aug 07, 2020

Last Modified: Aug 24, 2020

PROTOCOL integer ID: 40174
Sarah J Reiling

McGill University

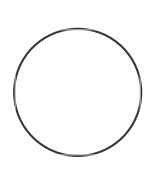




\section{ABSTRACT}

\section{How the Nextera DNA Flex Assay Works}

The Nextera DNA Flex library prep kit uses a bead-based transposome complex to tagment genomic DNA, which is a process that fragments DNA and then tags the DNA with adapter sequences in one step. After it is saturated with input DNA, the bead-based transposome complex fragments a set number of DNA molecules. This fragmentation provides flexibility to use a wide DNA input range to generate normalized libraries of consistent tight fragment size distribution. Following tagmentation, a limited-cycle PCR adds Nextera DNA Flex-specific index adapter sequences to the ends of a DNA fragment. This step enables capability across all Illumina sequencing platforms. A subsequent Sample Purification Beads (SPB) cleanup step then purifies libraries for use on an Illumina sequencer. The doublestranded DNA library is denatured before hybridization of the biotin probe oligonucleotide pool.

\section{PCR Amplicons for Nextera Flex}

When starting with PCR amplicons, the PCR amplicon must be $>150 \mathrm{bp}$. The standard clean up protocol depletes libraries $<500 \mathrm{bp}$. Therefore, Illumina recommends that amplicons $<500$ bp undergo a $1.8 \times$ sample purification bead volume ratio to supernatant during Clean Up Libraries on page 11. Shorter amplicons can otherwise be lost during the library cleanup step. Tagmentation cannot add an adapter directly to the distal end of a fragment, so a drop in sequencing coverage of $\sim 50 \mathrm{bp}$ from each distal end is expected. To ensure sufficient coverage of the amplicon target region, design primers to extend beyond the target region by $50 \mathrm{bp}$ per end.

More information can be found here:

https://emea.support.illumina.com/content/dam/illuminasupport/documents/documentation/chemistry_documentation/samplepreps_nexter a/nextera_dna_flex/nextera-dna-flex-library-prep-reference-guide-100000002541607.pdf

\section{Amplicon Quantification and normalisation}

1 Use the 30 ul with 150 ng DNA for Nextera Flex Tagmentation.

Protocol can be found here:

https://emea.support.illumina.com/content/dam/illumina-

support/documents/documentation/chemistry_documentation/samplepreps_nextera/nextera_d na_flex/nextera-dna-flex-library-prep-reference-guide-1000000025416-07.pdf

Start on page 7 with the Tagmentation. 


\section{Tagment Genomic DNA}

\section{Tagment Genomic DNA}

This step uses the Bead-Linked Transposomes (BLT) to tagment DNA, which is a process that fragments and tags the DNA with adapter sequences.

\section{Consumables}

Bead-Linked Transposomes (BLT)

> Tagmentation Buffer 1 (TB1)

- Nuclease-free water

- 96-well PCR plate

- Microseal 'B' adhesive seal

- $1.7 \mathrm{ml}$ microcentrifuge tubes

8-tube strip

1 Pipette tips

- $20 \mu \mathrm{l}$ multichannel pipettes

> $200 \mu \mathrm{l}$ multichannel pipettes

\section{About Reagents}

- BLT must be stored at temperatures above $2^{\circ} \mathrm{C}$. Do not use BLT that has been stored below $2^{\circ} \mathrm{C}$.

\section{Preparation}

1 Prepare the following consumables:

\begin{tabular}{lll} 
Item & Storage & Instructions \\
\hline BLT & $2^{\circ} \mathrm{C}$ to $8^{\circ} \mathrm{C}$ & Bring to room temperature. Vortex to mix. Do not centrifuge before pipetting. \\
\hline TB1 & $-25^{\circ} \mathrm{C}$ to $-15^{\circ} \mathrm{C}$ & Bring to room temperature. Vortex to mix. \\
\hline
\end{tabular}

2 Save the following TAG program on the thermal cycler:

- Choose the preheat lid option and set to $100^{\circ} \mathrm{C}$

- Set the reaction volume to $50 \mu \mathrm{l}$

- $55^{\circ} \mathrm{C}$ for 15 minutes

- Hold at $10^{\circ} \mathrm{C}$

\section{Procedure}

1 Add 2-30 $\mu$ l DNA to each well of a 96-well PCR plate so that the total input amount is 100-500 ng.

2 If DNA volume $<30 \mu$ l, add nuclease-free water to the DNA samples to bring the total volume to $30 \mu$.

3 Vortex BLT (yellow cap) vigorously for 10 seconds to resuspend. Repeat as necessary.

4 Combine the following volumes to prepare the tagmentation master mix. Multiply each volume by the number of samples being processed.

$\rightarrow \operatorname{BLT}(11 \mu \mathrm{l})$

$>\operatorname{TB} 1(11 \mu \mathrm{l})$

Reagent overage is included in the volume to ensure accurate pipetting. 
5 Vortex the tagmentation master mix thoroughly to resuspend.

6 Divide the tagmentation master mix volume equally into an 8-tube strip.

7 Using a $200 \mu$ multichannel pipette, transfer $20 \mu$ lagmentation master mix to each well of the plate containing a sample. Use fresh tips for each sample column.

8 Discard the 8-tube strip after the tagmentation master mix has been dispensed.

9 Pipette each sample 10 times to resuspend. Use fresh tips for each sample column.

10 Seal the plate with Microseal 'B', place on the preprogrammed thermal cycler, and run the TAG program.

Note

For automated protocol : at step 7, 22 ul tagmentation master mix is transfered.

\section{Post Tagmentation Cleanup}


This step washes the adapter-tagged DNA on the BLT before PCR amplification.

\section{Consumables}

> Tagment Stop Buffer (TSB)

- Tagment Wash Buffer (TWB)

- 96-well plate magnet

> Microseal 'B' adhesive seal

$>$ Pipette tips

> $20 \mu$ l multichannel pipettes

- $200 \mu$ multichannel pipettes

\section{Preparation}

1 Prepare the following consumables:

\begin{tabular}{lll}
\hline Item & Storage & Instructions \\
\hline TSB & $15^{\circ} \mathrm{C}$ to $30^{\circ} \mathrm{C}$ & $\begin{array}{l}\text { If precipitates are observed, heat at } 37^{\circ} \mathrm{C} \text { for } 10 \text { minutes, and then vortex until } \\
\text { precipitates are dissolved. Use at room temperature. }\end{array}$ \\
\hline TWB & $15^{\circ} \mathrm{C}$ to $30^{\circ} \mathrm{C}$ & Use at room temperature. \\
\hline
\end{tabular}

2 Save the following PTC program on the thermal cycler:

- Choose the preheat lid option and set to $100^{\circ} \mathrm{C}$

- Set the reaction volume to $60 \mu \mathrm{l}$

- $37^{\circ} \mathrm{C}$ for 15 minutes

r Hold at $10^{\circ} \mathrm{C}$

\section{Procedure}

1 Add $10 \mu \mathrm{l}$ TSB to the tagmentation reaction.

2 Slowly pipette each well 10 times to resuspend the beads.

3 Seal the plate with Microseal 'B', place on the preprogrammed thermal cycler, and run the PTC program.

4 Place the plate on the magnetic stand and wait until liquid is clear ( 3 minutes).

5 Using a multichannel pipette, remove and discard supernatant.

6 Wash two times as follows:

a Remove the sample plate from the magnetic stand and use a deliberately slow pipetting technique to add $100 \mu \mathrm{l}$ TWB directly onto the beads. A deliberately slow pipetting technique minimizes the potential of TWB foaming to avoid incorrect volume aspiration and incomplete mixing.

b Pipette slowly until beads are fully resuspended.

c Place the plate on the magnetic stand and wait until the liquid is clear ( 3 minutes).

d Using a multichannel pipette, remove and discard supernatant.

7 Remove the plate from the magnetic stand and use a deliberately slow pipetting technique to add $100 \mu \mathrm{l}$ TWB directly onto the beads.

8 Pipette each well slowly to resuspend the beads.

9 Seal the plate and place on the magnetic stand until the liquid is clear ( $\sim 3$ minutes). Keep on the magnetic stand until step 4 of the Procedure section in Amplify Tagmented DNA.

The TWB remains in the wells to prevent overdrying of the beads.

\section{Pool Libraries}




\section{Pool Libraries}

When the DNA input is 100-500 ng, quantifying and normalizing individual libraries generated in the same experiment is not necessary. However, the final yield of libraries generated in separate experiments can vary slightly.

To achieve optimal cluster density, pool equal library volumes and quantify the pool before sequencing.

\section{DNA Inputs of $100-500$ ng}

1 Combine $5 \mu$ l of each library (up to 384 libraries) in a $1.7 \mathrm{ml}$ microcentrifuge tube.

2 Vortex to mix, and then centrifuge.

3 Quantify the library pool using a dsDNA fluorescent dye method, such as Qubit or PicoGreen.

\section{For DNA Inputs of $<100 \mathrm{ng}$}

1 Quantify each library individually using Qubit or PicoGreen.

\section{Amplify Tagmented DNA}

Amplify Tagmented DNA

This step amplifies the tagmented DNA using a limited-cycle PCR program. The PCR step adds Index 1 (i7) adapters, Index 2 (i5) adapters, and sequences required for sequencing cluster generation. To confirm the indexes selected for low plexity pooling have the appropriate color balance, see the Index Adapters Pooling Guide (document \# 1000000041074).

Index adapter tubes or plates are ordered separately from the library prep components. For a list of compatible index adapters for use with this protocol, see Kit Contents on page 24.

\section{Consumables}

- Enhanced PCR Mix (EPM)

- Index adapters (tubes or plate)

- Nuclease-free water

- Microseal 'B' adhesive seal

$1.7 \mathrm{ml}$ microcentrifuge tubes

$\checkmark$ Pipette tips

> $20 \mu$ multichannel pipettes

> $200 \mu$ multichannel pipettes

\section{About Reagents}

- Index adapter plates

- A well may contain $>10 \mu$ l of index adapters.

- Do not add samples to the index adapter plate.

- Each well of the index plate is single use only.

- Index adapter tubes

- Open only one index adapter tube at a time to prevent misplacing caps. Alternatively, use fresh caps after opening each tube. 


\section{Preparation}

1 Prepare the following consumables:

\begin{tabular}{lll}
\hline Item & Storage & Instructions \\
\hline EPM & $-25^{\circ} \mathrm{C}$ to $-15^{\circ} \mathrm{C}$ & Thaw on ice. Invert to mix, then briefly centrifuge. \\
\hline Index Adapters & $-25^{\circ} \mathrm{C}$ to $-15^{\circ} \mathrm{C}$ & $\begin{array}{l}\text { Thaw at room temperature. } \\
\text { [Tubes] Vortex to mix, then centrifuge briefly. } \\
\text { [Plates] Spin briefly before use. }\end{array}$ \\
\hline
\end{tabular}

2 Save the following BLT PCR program on a thermal cycler using the appropriate number of PCR cycles, indicated in the table below.

- Choose the preheat lid option and set to $100^{\circ} \mathrm{C}$

- $68^{\circ} \mathrm{C}$ for 3 minutes

- $98^{\circ} \mathrm{C}$ for 3 minutes

- $(\mathrm{X})$ cycles of:

- $98^{\circ} \mathrm{C}$ for 45 seconds

1 $62^{\circ} \mathrm{C}$ for 30 seconds

- $68^{\circ} \mathrm{C}$ for 2 minutes

- $68^{\circ} \mathrm{C}$ for 1 minutes

Hold at $10^{\circ} \mathrm{C}$

\begin{tabular}{cc} 
Total DNA Input (ng) & Number of PCR Cycles (X) \\
\hline $1-9$ & 12 \\
\hline $10-24$ & 8 \\
\hline $25-49$ & 6 \\
\hline $50-99$ & 5 \\
\hline $100-500$ & 5 \\
\hline Blood/Saliva & 5
\end{tabular}

\section{Procedure}

1 Combine the following volumes to prepare the PCR master mix. Multiply each volume by the number of samples being processed.

$\rightarrow \operatorname{EPM}(22 \mu \mathrm{l})$

- Nuclease-free water $(22 \mu \mathrm{l})$

Reagent overage is included in the volume to ensure accurate pipetting.

2 Vortex, and then centrifuge the PCR master mix at $280 \times \mathrm{g}$ for 10 seconds.

3 With the plate on the magnetic stand, use a $200 \mu$ multichannel pipette to remove and discard supernatant.

Foam that remains on the well walls does not adversely affect the library.

4 Remove from the magnet.

5 Immediately add $40 \mu$ PCR master mix directly onto the beads in each sample well. 
6 Immediately pipette to mix until the beads are fully resuspended. Alternatively, seal the plate and use a plate shaker at 1600 rpm for 1 minute.

7 Seal the sample plate and centrifuge at $280 \times \mathrm{g}$ for 3 seconds.

8 Add the appropriate index adapters to each sample.

\begin{tabular}{lll}
\hline Index Kit Type & Kit Configuration & Volume of Index Adapter per Sample \\
\hline 24 plex (dual index) & Individual tubes & $5 \mu \mathrm{l} \mathrm{i7}$ adapter \\
& & $5 \mu \mathrm{l}$ i5 adapter \\
\hline 96 plex (dual index) & 96-well plate & $10 \mu \mathrm{l}$ pre-paired i7 and i5 index adapters \\
\hline
\end{tabular}

9 Using a pipette set to $40 \mu$, pipette 10 times to mix. Alternatively, seal the plate and use a plate shaker at 1600 rpm for 1 minute.

10 Seal the plate with Microseal ' $\mathrm{B}$ ', and then centrifuge at $280 \times \mathrm{g}$ for 30 seconds.

11 Place on the thermal cycler and run the BLT PCR program.

\section{SAFE STOPPING POINT}

If you are stopping, store at $2^{\circ} \mathrm{C}$ to $8^{\circ} \mathrm{C}$ for up to 3 days.

Note

For automated protocol : at step 9, 44 ul tagmentation master mix is transfered.

This step uses double-sided bead purification procedure to purify the amplified libraries.

\section{Consumables}

- Sample Purification Beads (SPB)

- Resuspension Buffer (RSB)

- Freshly prepared $80 \%$ ethanol $(\mathrm{EtOH})$

- 96-well $0.8 \mathrm{ml}$ Polypropylene Deepwell Storage Plate (midi plate) (2)

96-well PCR plate

1 Microseal 'B' adhesive seal

- Microseal ' $\mathrm{F}$ ' foil seal

> $1.7 \mathrm{ml}$ microcentrifuge tubes

Nuclease-free water

\section{About Reagents}

- Sample Purification Beads

- Must be at room temperature before use

V Vortex before each use

- Vortex frequently to make sure that beads are evenly distributed

- Aspirate and dispense slowly due to the viscosity of the solution

\section{Preparation}

1 Prepare the following consumables: 


\begin{tabular}{lll}
\hline Item & Storage & Instructions \\
\hline SPB & $2^{\circ} \mathrm{C}$ to $8^{\circ} \mathrm{C}$ & Let stand at room temperature for 30 minutes. Vortex and invert to mix. \\
\hline RSB & $-25^{\circ} \mathrm{C}$ to $-15^{\circ} \mathrm{C}$ & Thaw and bring to room temperature. Vortex to mix. \\
\hline
\end{tabular}

2 Prepare fresh $80 \% \mathrm{EtOH}$ from absolute ethanol.

\section{Procedure}

1 Centrifuge at $280 \times \mathrm{g}$ for 1 minute to collect contents at the bottom of the well.

2 Place the plate on the magnetic stand and wait until the liquid is clear ( $\sim 5$ minutes).

3 Transfer $45 \mu$ l supernatant from each well of the PCR plate to the corresponding well of a new midi plate.

4 Vortex and invert SPB multiple times to resuspend.

5 For standard DNA input, perform the following steps.

a Add $40 \mu$ nuclease-free water to each well containing supernatant.

b Add $45 \mu \mathrm{l} \mathrm{SPB}$ to each well containing supernatant.

c Pipette each well 10 times to mix. Alternatively, seal the plate and use a plate shaker at $1600 \mathrm{rpm}$ for 1 minute.

d Seal the plate and incubate at room temperature for 5 minutes.

e Place on the magnetic stand and wait until the liquid is clear ( $\sim 5$ minutes).

f During incubation, thoroughly vortex the SPB (undiluted stock tube), and then add $15 \mu$ to each well of a new midi plate.

g Transfer $125 \mu \mathrm{l}$ supernatant from each well of the first plate into the corresponding well of the second plate (containing $15 \mu$ l undiluted SPB).

$\mathrm{h}$ Pipette each well in the second plate 10 times to mix. Alternatively, seal the plate and use a plate shaker at $1600 \mathrm{rpm}$ for 1 minute.

i Discard the first plate.

6 For small PCR amplicon input, perform the following steps.

a Add $81 \mu \mathrm{l}$ SPB to each midi plate well containing supernatant.

b Pipette each well 10 times to mix. Alternatively, seal the plate and use a plate shaker at $1600 \mathrm{rpm}$ for 1 minute.

7 Incubate the sealed midi plate at room temperature for 5 minutes.

8 Place on the magnetic stand and wait until the liquid is clear ( 5 minutes).

9 Without disturbing the beads, remove and discard supernatant.

10 Wash two times as follows.

a With the plate on the magnetic stand, add $200 \mu \mathrm{l}$ fresh $80 \% \mathrm{EtOH}$ without mixing.

b Incubate for 30 seconds.

c Without disturbing the beads, remove and discard supernatant.

11 Use a $20 \mu$ pipette to remove and discard residual EtOH.

12 Air-dry on the magnetic stand for 5 minutes.

13 Remove from the magnetic stand.

14 Add $32 \mu \mathrm{RSB}$ to the beads.

15 Pipette to resuspend.

16 Incubate at room temperature for 2 minutes.

17 Place the plate on the magnetic stand and wait until the liquid is clear ( 2 minutes).

18 Transfer $30 \mu$ l supernatant to a new 96-well PCR plate.

\section{SAFE STOPPING POINT}

If you are stopping, seal the plate with Microseal ' $\mathrm{B}$ ' adhesive or Microseal ' $\mathrm{F}$ ' foil seal, and store at $-25^{\circ} \mathrm{C}$ to $-15^{\circ} \mathrm{C}$ for up to 30 days. 
Note

At step 14, beads can be resuspended in 62 uL RSB, followed by a $60 \mathrm{uL}$ transfer of supernatant at step 18. This is used to decrease the concentration of the final pool in order to facilitate the QC step.

\section{Check Library Quality}

\section{$7 \quad$ Check Library Quality (Optional)}

1 Run $1 \mu$ l library or pooled libraries on one of the following instruments:

- Advanced Analytical Fragment Analyzer with the HS-NGS High Sensitivity 474 kit. - Add $1 \mu \mathrm{l}$ RSB to the library to achieve the $2 \mu \mathrm{l}$ volume required for Fragment Analyzer.

- Agilent 2100 Bioanalyzer with a High Sensitivity DNA kit.

\section{Dilute Libraries to the Starting Concentration}


This step dilutes libraries to the starting concentration for your sequencing system and is the first step in a serial dilution. After diluting to the starting concentration, libraries are ready to be denatured and diluted to the final loading concentration.

For sequencing, Illumina recommends the read lengths indicated in the table below.

Table 2 Recommended Read Length on Illumina Systems

\begin{tabular}{lc} 
Sequencing System & $\begin{array}{l}\text { Read } \\
\text { Length }\end{array}$ \\
\hline $\begin{array}{l}\text { NovaSeq } 6000, \text { HiSeq } X^{\star} \text {, HiSeq } 3000 \text { and HiSeq 4000, NextSeq } 500 \text { and NextSeq 550, MiSeq, MiniSeq, } \\
\text { iSeq } 100\end{array}$ & $2 \times 151$ \\
\hline HiSeq 2000, HiSeq 2500 (high output) & $2 \times 126$ \\
\hline HiSeq 2500 (rapid run) & $2 \times 101^{* *}$ \\
\hline
\end{tabular}

*Not compatible with IDT for Illumina Nextera DNA UD Indexes (10bp)

${ }^{* *}$ Assumes the use of the 200 cycle kit

IDT for Illumina Nextera DNA UD Indexes uses 10 base pair index codes that differ from the Nextera DNA CD Indexes, and which use 8 base pair index codes. This change in base pair index codes can require adjustments to your sequencing run set up.

1 Calculate the molarity value of the library or pooled libraries using the following formula.

- For libraries qualified on a Bioanalyzer, use the average size obtained for the library.

- For all other qualification methods, use $600 \mathrm{bp}$ as the average library size.

$\frac{n g / \mu l \times 10^{6}}{660 \frac{g}{m o l} \times \text { average library size }(b p)}=$ Molarity $(n M)$

2 Using the molarity value, calculate the volumes of RSB and library needed to dilute libraries to the starting concentration for your system.

\begin{tabular}{|c|c|c|}
\hline Sequencing System & $\begin{array}{l}\text { Starting Concentration } \\
\text { (nM) }\end{array}$ & Final Loading Concentration ( $\mathrm{pM}$ ) \\
\hline HiSeq 2500 and HiSeq 2000 (high output modes) & 2 & 12 \\
\hline HiSeq 2500 (rapid run mode) & 2 & 8.5 \\
\hline HiSeq X, HiSeq 4000, and HiSeq 3000 & $2-3$ & $200-300$ \\
\hline iSeq 100 & 2 & 200 \\
\hline MiniSeq & 2 & $1.2-1.3$ \\
\hline MiSeq (v3 reagents) & 4 & 12 \\
\hline NextSeq 550 and NextSeq 500 & 2 & $1.2-1.3$ \\
\hline NovaSeq 6000 & 2 & $\begin{array}{c}\text { See document \# } 1000000019358 \\
\text { (NovaSeq } 6000 \text { System Guide) }\end{array}$ \\
\hline
\end{tabular}

3 Dilute libraries using RSB:

- Libraries quantified as a multiplexed library pool-Dilute the pool to the starting concentration for your system.

- Libraries quantified individually-Dilute each library to the starting concentration for your system. Add $10 \mu \mathrm{l}$ each diluted library to a tube to create a multiplexed library pool.

4 Follow the denature and dilute instructions for your system to dilute to the final loading concentration.

- For the iSeq 100 System, see the system guide for dilution instructions (libraries are automatically denatured).

- For the NovaSeq 6000 System, see the system guide for pool and denature instructions.

- For the HiSeq 4000 and HiSeq 3000 Systems, see the cBot 2 or cBot system guide for reagent preparation instructions.

- For all other systems, see the denature and dilute libraries guide.

The final loading concentrations are a starting point and general guideline. Optimize concentrations for your workflow and quantification method over subsequent sequencing runs or by flow cell titration. 
